\title{
ASSESSING SELF-ESTEEM AND PERCEIVED PHYSICAL COMPETENCE IN ELDERLY USING THE PHYSICAL SELF-PERCEPTION PROFILE
}

\author{
José P. Ferreira ${ }^{1}$, Ana M. Teixeira ${ }^{1}$, Alain G. Massart ${ }^{1}$, Edith Filaire ${ }^{2}$, \\ 1. University of Coimbra, Portugal \\ 2. University of Orléans, France
}

The paper examined the psychometric properties of the Physical Self-Perception Profile (PSPP) when used with elderly groups and described gender differences in physical self and global self-esteem. A sample of 207 elderly participants (age 65-93 years, $\mathrm{M}=76.54$ and SD=6.56) completed the PSPP and the Rosenberg Self-esteem Scale as a self-reported measure of physical self and global self-esteem during different social and cultural activities in senior daily care centres. Reliability, factor structure, hierarchical structure and criterion-related validity were assessed. Findings revealed limited gender differences for the total sample, with males expressing more positive feelings than females for physical strength $(\mathrm{p}<.05)$, however when analysed by age group, statistical differences were found for physical confidence $(\mathrm{p}<.05)$, body $(\mathrm{p}<.05)$ and physical self-worth $(\mathrm{p}<.05)$ with females reporting more positive values than males. Factor analysis results showed limited goodness-of-fit indexes for the model revealing structural problems for the use of PSPP with elderly groups. Elderly participants may perceive themselves in the physical domain using different feelings than those extensively assessed with the PSPP in younger groups highlighting the hypothetical change in self-perceptions over the lifespan.

Keywords: physical self, self-esteem, elderly, PSPP, psychometric properties

\section{INTRODUCTION}

Aging is often associated with declines in health, with important decrease in muscle mass, strength, endurance, and a continuous decrease both in physical and cognitive function that can have significant impact on an individual's ability to perform daily life activities and independence (McAuley, Konopack, Motl, Morris, Doerksen, \& Rosengren, 2006). The aging process leads not only to an important decline in functional capacity (Rickli \& Jones, 2001) but also in physical and social well-being with negative implications for mental health and well-being (Fox, Stathi, McKenna, \& Davis, 2007).

In the last decade health organizations have emphasised self-esteem as a central component of mental health (U.S. Department of Health and Human Services, 2001) and it has been widely accepted as an important indicator of emotional stability, adjustment to life demands and therefore mental health (Fox, 2000). Selfesteem is often regarded as the single most important contributor to mental health and an important aspect of quality of life, thereby being considered as a possible target for public health campaigns (Department of Health, 2004; NIH Seniors Health, 2010).

Positive self-esteem is seen as a basic feature of mental health, but also as a protective factor that contributes to better health and positive social behaviour through its role as a buffer against the impact of negative influences (Mann, Hosman, Schaalma, \& de Vries, 2004). Meanwhile low self-esteem is often associated with poor health behaviours and mental disorders (Fox, 2000; Mann et al., 2004) such as depression (Mirabel-Sarron, Vera, \& Samuel-Lajeunesse, 2001), anxiety (Silverstone, 2011), anorexia and bulimia (Button, Sonuga-Barke, Davies, \& Thompson, 2011) substance abuse (Emery, McDermott, Holcomb, \& Marty, 2009) and violence and high-risk behaviours (Ostrowsky, 2010).

Because of the global nature of self-esteem and its potential to be influenced by interactions in several life domains, researchers have consistently emphasised the need to assess several dimensions of the self, alongside with self-esteem (Fox, 1990; Marsh, Richards, Jonhson, Roche, \& Tremayne, 1994). The physical self has emerged as consistently important, demonstrating moderately strong 
correlations with self-esteem (.50-.70) throughout the lifespan (Fox, 2000). Additionally, physical self-perceptions have been directly related to indicators of emotional adjustment, independent of self-esteem (Sonstroem \& Potts, 1996).

In spite of the important contribution of self-esteem and the physical self as mental health indicators and as predictors of positive emotional adjustments, little investigation has been conducted about how middle age and old adults evaluate their feelings in the physical domain (Gothe, Mullen, Wójcicki, Mailey, White, Olson, Szabo, Kramer, \& McAuley, 2011; Opdenacker, Delecluse \& Boen, 2009; Sonstroem, Speliotis, \& Fava, 1992; Semerjian $\&$ Stephens, 2007) requiring further research to understand the development of specific individual feelings among older people.

Semerjian and Stephens (2007) assessed the physical self, functional fitness and physical activity of 102 community women ( $M=75.97$; $\mathrm{SD}=7.33$ ) living in USA and reported a moderate relationships between perceived functional ability and perceived health, between perceived functional ability and overall physical self-worth, and between perceived health and overall physical selfworth. Participants who used temporal comparison when evaluating their abilities tend to present higher levels of perceived functional ability. These authors also reported that younger women had higher self-perceptions of health than older women. In this particular group perceptions of health seemed to be more susceptible to individual's perception that, with aging, health declines than perceptions of attractiveness, functional ability or overall physical self-worth. Another interesting finding from this study is that participant's evaluations of decline or improvements in their ability, over time, were not significant to their current evaluation of their abilities, suggesting that those who feel they have declined over time did not present lower self-perceptions as one might expect.

Opdenacker, et al. (2009) assessed the impact of physical activity and exercise on 235 community adults in Leuven, Belgium and reported significant positive effects on physical self-perceptions and self-esteem in older adults, participating in a lifestyle physical activity programme $(\mathrm{M}=66.30 ; \mathrm{SD}=3.99)$ and in a structured exercise intervention programme $(\mathrm{M}=66.99 ; \mathrm{SD}=4.32)$, both in the short and long term. The lifestyle group showed significant short-term improvements in physical condition, sport competence, body attractiveness and physical self-worth. In the structured exercise group, there were significant short-term effects on physical condition and sport competence. With respect to long-term effects, the lifestyle program participants had significant effects on body attractiveness and global self-esteem and the structured exercise intervention had long-term effects on physical condition, sport competence and body attractiveness.

The purpose of the present study is twofold. The major aims are: i) to test the psychometric properties of the PSPP and it's adequacy to be used with elderly participants. Recently Opdenacker, et al. (2009) used confirmatory factor analysis to assess the goodness of fit of a revised version of the EXSEM model (Sonstroem \& Morgan, 1989), linking physical self-perceptions and global selfesteem to exercise intervention and to selfefficacy with older participants but did not analyse the specific structure of the instrument and ii) to describe self-esteem and physical competence of elderly participants as well as to analyse hypothetical gender and age differences between groups.

Thus we examined the following specific research questions: a) Is PSPP a reliable and valid instrument to assess physical selfperceptions in elderly groups? b) What is the relationship between physical self-perceptions and global self-esteem in elderly participants? c) Are there any significant gender and age differences among elderly participants?

\section{METHOD}

\section{Participants}

The PSPP was administrated to a sample of 207 cognitively healthy elderly participants (no cognitive degenerative disease was diagnosed by GP's) aged 65 to 93 years old, 105 males $(\mathrm{M}=76.63, \quad \mathrm{SD}=6.58)$ and 102 females $(\mathrm{M}=76.45, \mathrm{SD}=6.57)$ living in Centre Portugal. Participants were recruited from private and public sector social organizations or were retired but participating in social and cultural activities in senior daily care centres in both rural and urban settings aiming to reflect the academic and cultural diversity of the adult population in this geographic area. An effort was made to recruit participants from different 
backgrounds and life experiences in order to assure the representativeness of the target population.

\section{MEASUREMENTS}

\section{Physical Self-Perception Profile}

The Physical Self-Perception Profile consists of four sub-scales (physical confidence, body, strength and physical selfworth) with six items displayed in a structured alternative format. Respondents are presented with two contrasting descriptions of people that are asked to select the description most like themselves and afterwards to express the intensity of the agreement with that description, i.e., to choose whether this description is "sort of true to me" or "really true to me". This structured alternative format was developed by Harter (1985) and has been shown to overcome socially desirable responding (Fox, 1990), scoring from 1 to 4 each one of the six items in each subscale with a possible range of scores from 6 to 24 .

The psychometric properties of the PSPP were presented by Fox (1990) and replicated in many studies over the years. In Fox's original studies internal consistency Cronbach Alpha coefficients for males and females ranged from .81 to .92 and test-retest stability coefficients ranged from $\mathrm{r}=.74$ to .92 over a 16-day period and between $\mathrm{r}=.81$ and .88 over a 23-day period. Chase (1991) tried to adapt the PSPP into a measure that was more relevant to an aging population and developed a version for adults reporting a relationship between physical self-perceptions and the amount and type of activities in which older adults choose to participate. This version was identified as measuring four subdomains: sport competence, appearance, functional capacity and health/disease state apart from physical selfworth, however little information is available about it's final structure, validity and reliability.

The psychometric properties of the Portuguese version of the Physical Self Perception Profile were initially presented by Fonseca and Fox (2002) and confirmed by Ferreira and Fox (2007) suggesting the adequacy of the instrument to be used with the Portuguese population. The factor structure of the Portuguese version is slightly different from the original and involves the combination of physical competence and physical condition into a unique factor - physical confidence based on conceptual and cultural reasons previously described by Ferreira and Fox (2007) and Van der Vliet, Knapen, Onghena, Fox, VanCoppenolle, David, Pieters, and Peuskens (2002) for non-English speaking cultures with good validity and reliability results. In Ferreira and Fox's study, internal consistency alpha values ranged from .66 to .88 for females and .58 to .84 for males.

\section{Self-Esteem Scale}

The Rosenberg Self-Esteem Scale - RSES (Rosenberg, 1965) is a ten items unidimensional scale that uses a 4-point likert response format varying from strongly agree to strongly disagree. Self-esteem is represented by the sum of all item scores providing a possible range of 10 to 40 with higher scores indicating high Self-esteem. A previous study reported a test-retest stability coefficient of $r=.75$ (Ferreira \& Fox, 2007).

\section{PROCEDURE}

Participants were recruited through verbal announcements at private and social security institutions and senior daily care centres, among those who expressed their written volunteer participation. A letter was sent to the directors requesting permission for the participant's recruitment and staff cooperation. Participants were all cognitively sound and able to answer questions independently. No incentives were provided. The purpose of the study was explained and participants were tested individually due to the complexity of the instruments.Questionnaires were administrated using standardised instructions by three research assistants that were available to answer any question during administration sessions. In some cases interview techniques were used among those with low academic levels or that expressed difficulty to understand some of the PSPP items. Research assistants received specific one-to-one interviewing training to avoid directing the participant's answers and ensure confidentiality. The time of administration was 15-40 minutes. Global data collection period lasted for two months. Test-retest stability for the PSPP and RSES was assessed through a re-administration of the instruments to a randomly selected sample $(n=30)$, i.e., a sub-sample of the original sample where every person has an equal 
chance of being chosen to take part in this subgroup (Bordens \& Abbott, 2008), after a lapse period of three weeks.

\section{Statistical analysis}

All analyses were conducted separately by gender. Differences have consistently emerged across studies and substantiate the need for self-perception analyses to be systematically conducted by gender (Fox, 1990; Ferreira \& Fox, 2007; Van der Vliet et al., 2002). The full range of descriptive statistics was calculated for all sub-domains of the PSPP and for RSES. An alpha level of .05 was used for all statistical tests. The normality of the population was confirmed using Kolmogorov-Smirnov Test. Internal consistency was measured using Cronbach Alpha coefficient values (Cronbach, 1951) and stability using Person's productmoment r. Bivariate and partial correlations, including zero-order correlation coefficients, were used to analyse the relationship between the different physical self subdomains as well as with global self-esteem. Stepwise multiple regression was used to assess the level of explained variance in the physical self-worth by the remaining PSPP subscale scores.

Validation of the PSPP was undertaken using confirmatory factor analyses (CFA). The fit of the model was assessed using multiple indexes, the chi-square $\left(\chi^{2}\right)$, the $\chi^{2} /$ df ratio value, which should be smaller than 3.0 , the comparative fit index (CFI), the goodness of fit index (GFI) and the incremental fit index (IFI), which should be larger than .90 and the standardized root mean square residuals (SRMR) which should be smaller than .08, for a good-fitting model (Hu \& Bentler, 1999). Comparison between groups was analysed using independent T-test.

\section{RESULTS}

\section{Descriptive data}

Table 1 provided mean score values (M) and standard deviations (SD) from male and female participants from different age groups in our study and from Sonstroem, Speliotis and Fava's (1992) study also with elderly participants.

\section{TABLE 1}

Means and standard deviations from PSPP sub-scales and global self-esteem by gender, age group and total sample

\begin{tabular}{|c|c|c|c|c|c|c|}
\hline \multirow[t]{2}{*}{ Sub Scales } & \multicolumn{4}{|c|}{ Present study } & \multicolumn{2}{|c|}{$\begin{array}{c}\text { Sonstroem, Speliotis \& Fava, } \\
1992\end{array}$} \\
\hline & \multicolumn{2}{|c|}{ (65-75 yrs.) } & \multicolumn{2}{|c|}{ (> 75 yrs.) } & & \\
\hline \multirow[t]{2}{*}{ Female } & \multicolumn{2}{|c|}{$\mathrm{n}=44$} & \multicolumn{2}{|c|}{$\mathrm{n}=58$} & \multicolumn{2}{|c|}{$\bar{n} \mathrm{n}=149$} \\
\hline & M & SD & M & SD & M & SD \\
\hline PhyConf & 15.27 & 3.86 & 12.81 & 4.17 & - & - \\
\hline Body & 17.20 & 3.37 & 15.14 & 3.92 & 14.82 & 4.50 \\
\hline Stren & 15.70 & 4.46 & 14.47 & 5.34 & 15.16 & 4.11 \\
\hline PSW & 18.05 & 3.65 & 15.95 & 4.29 & 15.75 & 4.30 \\
\hline GSE & 27.45 & 4.59 & 30.16 & 3.99 & 33.44 & 5.01 \\
\hline \multirow[t]{2}{*}{$\underline{\text { Male }}$} & \multicolumn{2}{|c|}{$\mathrm{n}=47$} & \multicolumn{2}{|c|}{$\mathrm{n}=58$} & \multicolumn{2}{|c|}{$\mathrm{n}=111$} \\
\hline & M & SD & M & SD & M & SD \\
\hline PhyConf & 13.23 & 4.54 & 14.24 & 4.68 & - & - \\
\hline Body & 15.68 & 4.00 & 16.19 & 3.81 & 15.86 & 3.86 \\
\hline Stren & 15.91 & 4.97 & 16.17 & 6.37 & 16.15 & 3.77 \\
\hline PSW & 16.09 & 4.08 & 15.84 & 4.06 & 16.89 & 3.66 \\
\hline GSE & 28.64 & 4.84 & 28.69 & 5.62 & 33.98 & 4.59 \\
\hline
\end{tabular}

PhyConf - Physical Confidence; Body - Body Attractiveness; Stren - Physical Strength; PSW - Physical Self Worth; GSE Global Self-Esteem. 
Results from table 1 showed a different pattern for the PSPP subscales with adult participants. Females presented higher values of body attractiveness and physical self-worth while males presented higher mean score values for physical strength, body attractiveness and physical self-worth. Descriptive values from males and females analysed by age group revealed that in the age group from 65-75 yrs. females presented higher mean score values for physical confidence, body and physical self-worth when compared with males while in the age group of 75 or more yrs. females presented higher mean score values for physical selfworth and global self-esteem than males. Finally, in the age group of more than 75 yrs. males reported higher mean score values for the majority of the sub-domains but no significant statistical differences were found for gender.

\section{Reliability}

Internal consistency and reliability was undertaken per gender using Cronbach Alpha coefficient values. Results revealed a good to excellent internal consistency with Alphas values of .81, .90 and .77 for physical confidence, strength and body in females and $.84, .94$ and .78 for physical confidence, strength and body in males. Test-retest stability revealed high Pearson's $r$ correlation coefficients for physical confidence, body and strength with $\mathrm{r}=.86, .96$ and .93 for female and $\mathrm{r}=.80, .92$ and .94 for male, for a 21-days lapse period. PSPP therefore demonstrated preliminary internal consistency as well as stability. Moreover, in the present study RSES also presented an acceptable test-retest stability value of $r=.71$ for females and $r=.74$ for males with a three weeks period between applications.

\section{Hierarchical structure}

Figure 1 presents Pearson's $r$ coefficients (zero-order and partial correlation coefficients) and multiple regression analysis values (in brackets) from female $(\mathrm{n}=102)$ and male $(\mathrm{n}=105)$ adult participants.

\section{FIGURE 1}
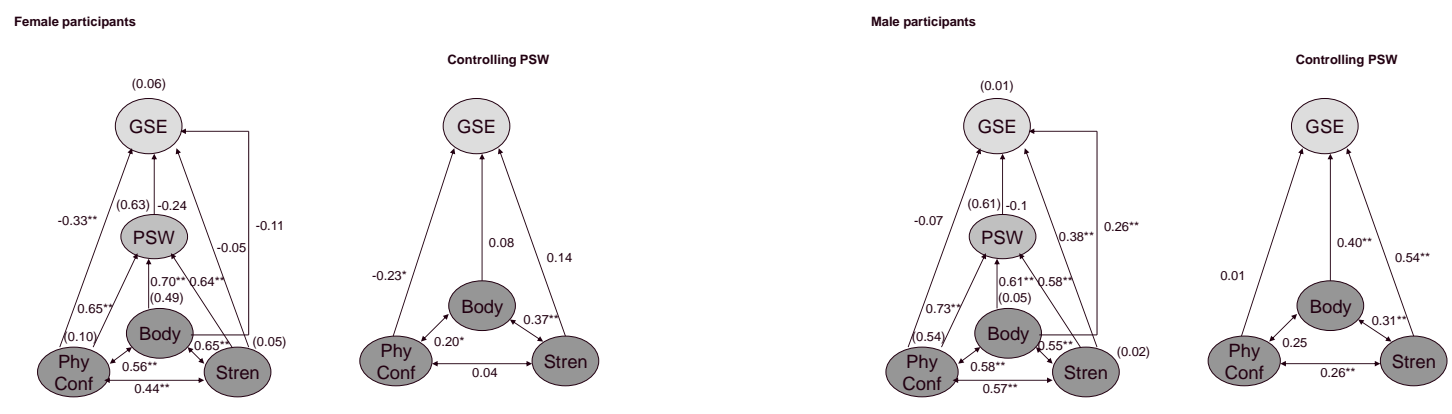

Pearson's r coefficients (zero-order and partial correlation coefficients) and multiple regression analysis values (in brackets) from female $(\mathrm{n}=102)$ and male $(\mathrm{n}=105)$ elderly participants. GSE - Global Self-Esteem; PSW Physical Self Worth; PhyConf - Physical Confidence; Body - Body Attractiveness and Stren - Physical Strength

Results revealed that no support was found for the hierarchical organization between constructs among adult groups. Physical selfworth did not show the strongest relationship with global self-esteem. Physical confidence in the female sub-group and body and strength in the male sub-group presented higher correlation values with global self-esteem than physical self-worth. In spite of the strong hierarchical organization found at the base of the model, with all subscales showing a stronger relationship with physical self-worth than with global self-esteem, the relationship between the three subscales and global selfesteem did not decrease or extinguished when the effects of physical self-worth were statistically removed, particularly in the male sub-group. Those relationships decreased in all sub-domains for the female sub-group, however did not extinguish while increased in male group for body and strength sub-domains. Therefore correlational analyses did not 
confirm the hierarchical structure of the instrument.

Multiple regression analyses revealed that body in females and physical confidence in males were able to explain $48.7 \%$ and $53.8 \%$ of the variance in physical self-worth for both groups. In both cases the additional amount of variance explained by other sub-domains is limited. In the female elderly group physical confidence and strength together were able to explain an additional $14.7 \%$ of the total variance for physical self-worth with a total value of $62.9 \%$ while in the male elderly group body and strength were able to explain an additional $6.7 \%$ of the total variance in physical self-worth with a total value of $60.5 \%$. Finally, the amount of variance explained by physical self-worth functioning as a mediator between the PSPP sub-domains and global self-esteem at the apex is very limited for both subgroups $6.0 \%$ and $1.0 \%$ respectively.

\section{Factorial analysis}

Confirmatory factor analysis (CFA) using a three factors six items per factor model was conducted to assess the goodness-of-fit for the PSPP in elderly groups. This model was previously used with success by Van de Vliet et al. (2002) and Ferreira and Fox (2007) in non-English speaking cultures. Table 2 presents CFA results from male and female elderly participants as well as from the total sample $(\mathrm{N}=207)$.

The $\chi^{2}$ values obtained for female (242.81) and male (266.18) sub-samples differ significantly from the independent model $(\mathrm{p}<.001) . \chi^{2} / \mathrm{df}$ value, a ratio value used to assess the model was lower than 3.0 for all sub-groups as well as for the total sample showing a good adjustment (Joreskog, 1969). The comparative fit index (CFI), the goodness of fit index (GFI) and the incremental fit index (IFI) analysed across the different sub-samples and the entire sample were $.88, .77$ and .88 for male, $.87, .80$ and .87 for female, and $.91, .85$, and .91 for the total sample. The analysed model of three latent variables showed a better goodness of fit for the entire sample than for any of the female and male sub-samples, however most of the goodness-of-fit indexes showed a value slightly below .90 considered to be indicative of an adequate model fit. On the other hand, standardized root mean square residuals (SRMR) values were higher than .08 for both male and female samples but not for the total sample and did not support the good model fit for both male and female sub-groups (Hu \& Bentler, 1999).

Results from the Multivariate Lagrange Multiplier Test (MLMT) using the present model with elderly participants indicated that the model would be improved if items were allowed to load in more than one factor. The model would be significantly improved by adding 4 new parameters in the total sample $\left(\chi^{2}\right.$ improvement $=34.793, \mathrm{p}<.01)$. Most notably, the results indicated that two items (12 - Some people do not usually have a high level of stamina and fitness but others always maintain a high level of stamina and fitness, $\chi^{2}=19.798$, $\mathrm{p}<.01$; and 22 - Some people feel extremely confident about their ability to maintain regular exercise and physical condition but others don't feel quite so confident about their ability to maintain regular exercise and physical condition, $\chi^{2}=6.973, p<.01$ ) should cross load into the physical strength scale and two other items $(13$ - Some people feel embarrassed by their bodies when it comes to wearing few clothes but others do not feel embarrassed by their bodies when it comes to wearing few clothes, $\chi^{2}=6.856, \mathrm{p}<.01 ; 19$ - Some people tend to lack confidence when it comes to their physical strength but others are extremely confident when it comes to their physical strength, $\chi^{2}=6.508, \mathrm{p}<.01$ ) should cross load into the physical confidence scale.

All the other parameters were errors and didn't exhibit an effective contribution to the improvement of the model as they are random. Indices of overall model fit therefore do not entirely support the three factor structure of sub-domains with six items specifically in female and male samples but provide an acceptable support with the total sample in elderly participants.

Table 3 presents comparison between groups for gender and age group. Independent T-test results showed significant statistical differences between male and female elderly participants (total sample) for strength $(\mathrm{p}<.05$; Cohen's $\mathrm{d}=.274$ ) with males expressing more positive feelings about their physical strength than females and significant statistical differences in the age group of 65-75 yrs. for physical confidence $(\mathrm{p}<.05$; Cohen's $\mathrm{d}=$ .460), body ( $\mathrm{p}<.05$; Cohen's $\mathrm{d}=-.411)$ and physical self-worth $(\mathrm{p}<.05$; Cohen's $d=-.506)$ 
with females reporting more positive values

than males.

\section{TABLE 2}

Results from confirmatory factor analysis by gender and total sample

\begin{tabular}{lccccccccc}
\hline \hline Gender & Sample size & $\chi^{2}$ & Df & $p$ & $\chi^{2} / \mathrm{Df}$ & CFI & GFI & IFI & SRMR \\
& & & & & & & & & \\
\hline \hline Female & 102 & 242.81 & 132 & $<.001$ & 1.839 & .87 & .80 & .87 & .093 \\
Male & 105 & 266.18 & 132 & $<.001$ & 2.017 & .88 & .77 & .88 & .082 \\
Total sample & 207 & 310.83 & 132 & $<.001$ & 2.355 & .91 & .85 & .91 & .074
\end{tabular}

$\overline{\chi^{2}-\text { Chi-square; Df - Degrees of freedom; } p-p \text {-value; CFI - Confirmatory Fit Index; GFI - Goodness of fit }}$ index; IFI - Incremental fit index; SRMR - Standardized root mean square residuals

\section{TABLE 3}

P-values and effect sizes from PSPP sub-scales and global self-esteem by gender and age group

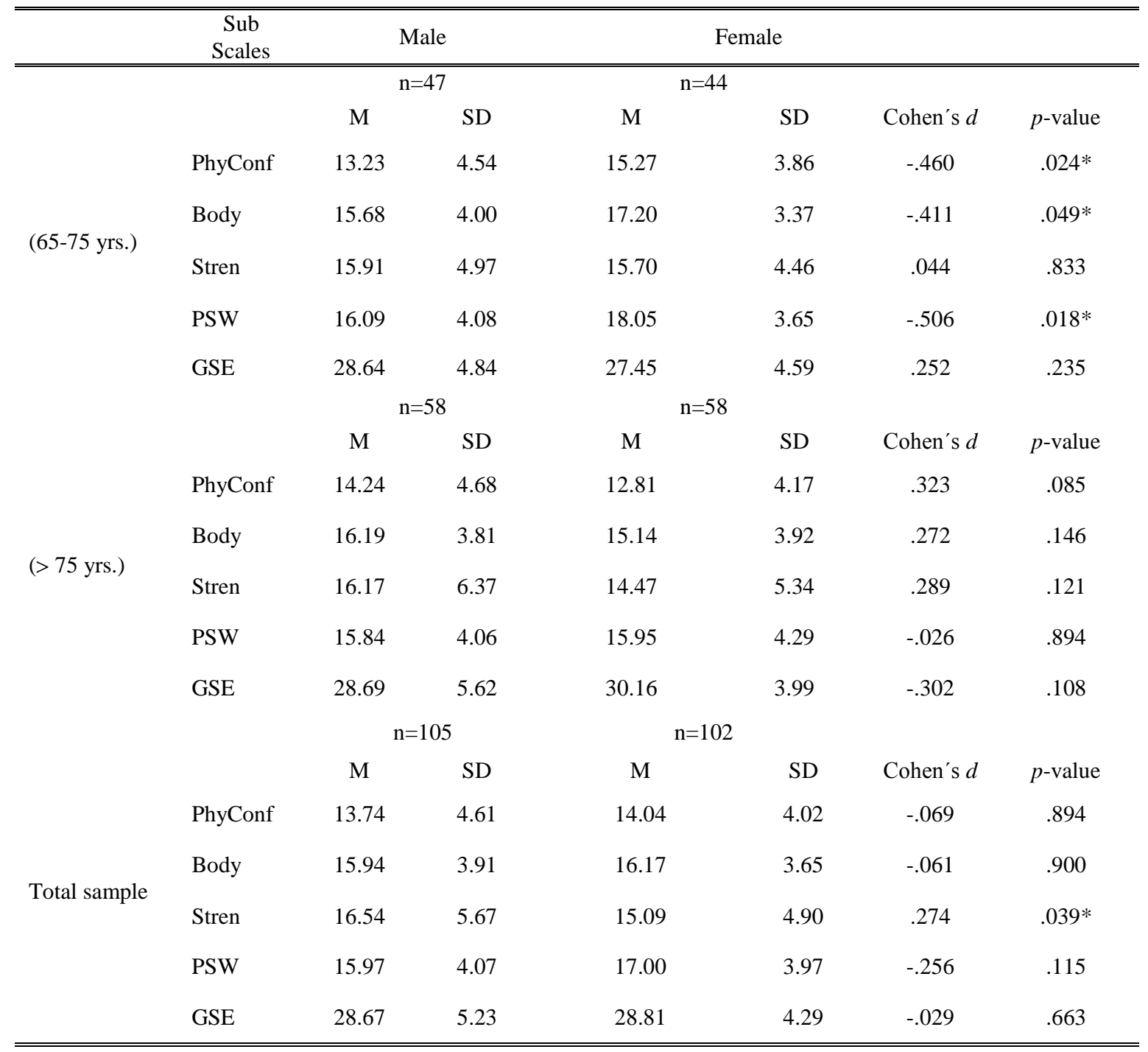

* Significant at $\mathrm{p}<0.05$

PhyConf - Physical Confidence; Body - Body Attractiveness; Stren - Physical Strength; PSW - Physical Self Worth; GSE - Global Self-Esteem 


\section{DISCUSSION}

The present study was designed to assess the psychometric properties of the PSPP with elderly groups and provide a comprehensive descriptive profile of old adult's self-esteem and physical self as well as to assess hypothetical age and gender differences between groups.

Descriptive results obtained in the present study are similar to those presented by Sonstroem, Speliotis and Fava (1992) using the same instrument in a middle age and older adult population ranging from 31 to $66 \mathrm{yrs}$. $(\mathrm{M}=44.1 ; \mathrm{SD}=11.6)$. In both studies physical self-worth and body attractiveness are very much valued among both male and female subgroups. PSW results are higher than those reported by Elavsky, McAuley. Molt, Konopack, Marquez, Hu, et al. (2005) using 174 elderly participants ranging from 60-75 years $(\mathrm{M}=66.7 ; \mathrm{SD}=5.35)$. Physical strength is also very much valued among elderly males, however present findings seem to reveal that along the life span, there might exist a progressive change on the individual level of perceptions among male and female elderly individuals. As times goes by old adult women may develop higher global levels of perception and satisfaction with their physical self and self-esteem as a result of a more positive and global view of their physical self rather than valuing particular domains, while old adult males value more particular dimensions of the self in particular those related with physical strength or body attractiveness. This hypothesis is partially supported by a recent study from Semerjian and Stephens (2007) that reported higher values of overall physical selfworth in women aged 65-99 yrs. that compare themselves favourably with others in their social comparison. Higher levels of physical self-worth accounted for more of the variance in self-perceptions $(24.8 \%)$ than the functional fitness predictors assessed (19.7\%) reflecting the importance of physical self-worth as a generalized outcome of perceptions from subdomain level and as a mediator between these sub-domains and the global self-esteem providing further evidence for it's importance in older women mental health and well-being.

The present study also reveals that the psychometric properties of the PSPP are not totally confirmed with elderly participants, discouraging it's full use as a reliable and valid instrument to assess physical self-perceptions in elderly groups. In spite of the good to excellent Cronbach Alpha reliability values obtained, the dimensionality and the hierarchical structure of the PSPP are quite poor and different from the ones described by Fox and Corbin (1989) and confirmed over the last three decades in several studies published with younger groups. These results are different from those recently reported by Opdenacker et al. (2009) using a sample of 186 elderly participants aged over 60 years $(\mathrm{M}=67.05 ; \mathrm{SD}=4.54)$, when assessing the goodness of fit of a revised version of the EXSEM model linking physical selfperceptions and global self-esteem to exercise intervention and to self-efficacy, in older participants. This inconsistency in the results may be explained by the existent age difference between the two samples used. The present sample is approximately 10 years older than the one used by Opdenacker et al. (2011) and as previously mentioned self-perceptions may change over time, in particular among elderly groups where functionality tends to decrease fast over time. A second reason that may explain the differences between the two studies is both the different APA background of the participants. In Opdenacker's et al. (2009) study participants included did not participate systematically in any endurance or strength training programme in the last 2 years preceding the study. They were not also physically active at moderate intensity for more than two hours per week at the time of recruitment. In the present study participants were mostly sedentary or participating in exercise recreational programmes at low to moderate intensity for less than two hours per week at time of recruitment.

At sub-domain level, body in females and physical confidence in males were able to explain $48.7 \%$ and $53.8 \%$ of the variance in physical self-worth for both groups, however the amount of variance explained by physical self-worth functioning as a mediator between the PSPP sub-domains and global self-esteem is very limited with $6.0 \%$ and $1.0 \%$ for both subgroups respectively. Fox and Corbin (1989) reported a value of $69 \%$ for female and $68 \%$ for male in young adults, however present limitations found in older participants are not unusual as they were previously reported in Sonstroem, Speliotis and Fava's (1992) study where physical self-worth served as an 
effective mediator between the sub-domains and self-esteem for strength and sport competence in females but totally failed to do so for attractive body and sport competence scales in males. Furthermore, these results are also in accordance with Ferreira and Fox (2007, 2008) when reporting very low $\mathrm{R}^{2}$ values and low negative correlations between physical self-worth and global self-esteem in other Portuguese groups such as adolescent, young adults and adults with physical impairment.

Further structural analysis, using confirmatory factor analysis with female and male groups as well as with the total sample, revealed a limited support for the use of PSPP with female and male elderly groups separately. However a better goodness of fit was revealed for the total sample with three out of four indexes showing a cut off value higher than .90 and with standardized root mean square residuals value lower than $.08(\mathrm{Hu} \mathrm{\&}$ Bentler, 1999).

Present results provide further evidence for the limitations previously suggested by Sonstroem, Speliotis and Fava (1992) about the factorial structure of PSPP when used with elderly participants. Based on exploratory factor analysis results (principal components analysis) these authors reported an overlap between physical self-worth and attractive body subscales in adults in their middle and later years due to a high number of crossloadings. This marked association may have been influenced by a perspective in elderly subjects that linked body evaluations with health concerns and subsequently with physical self-worth.

During the administration process several questions emerged about former sport competence items included in the physical confidence subscale which seemed to be quite problematic and source of confusion among elderly participants. In cultures such as the Portuguese, sport had for many years a limited tradition and impact in society due to the small number of opportunities provided, and for political reasons educational policies did not include for a long time sport as a priority (Ferreira \& Fox, 2007). Therefore citizens did not feel valued by their sport achievements, and older people showed great difficulty to understand this construct and to express their individual feelings about it as many of them never experienced any formal or informal involvement in sport activities over the life span.

Present results also seem to confirm that elderly participants may perceive themselves in a different way and are in accordance with the pioneering work of Chase (1991) when suggested the introduction of a functional capacity scale and a health/disease state scale to assess the physical self in adults, providing further evidence for the existence of alternative ways to influence the development of selfperceptions in the physical domain in elderly people. Individual feelings about physical confidence are much more related to endurance and stamina, including physical condition items rather than sport competence items as well as about present functional ability and health status. These results question, once again, the role of sport competence subscale in older adult's self-perceptions. This topic has been under discussion for quite a while and previous authors have already omitted in some studies the sport competence subscale of the PSPP because they did not consider it's relevance to the sedentary old adults population ( $\mathrm{Li}$, Harmer, Chaumeton, Duncan, \& Duncan, 2002; McAuley, Blissmer, Katula, Duncan, \& Mihalko, 2000; McAuley, Elavsky, Motl, Konopack, Hu, \& Marquez, 2005). In the present study many elderly participants expressed their physical competence through their perceived ability to do different daily tasks such as being able to walk, climb stairs and get out of bed independently as well as doing simple routines such as go shopping, cook a meal or prepare a cup of tea or coffee, rather than based on perceived ability in sport settings, i.e., elderly groups perceived physical confidence appears to be empowered through individual's perceptions of health-related reference rather than by performance issues.

Present results also seem to indicate a strong health concern with elderly participants actively pursuing health and functional goals and developing positive feelings about the functional dimension of their bodies as something very important for the way they perceived themselves and their perceived health status. This relationship between functionality and health is in accordance with the moderate relationship found by Semerjian and Stephens (2007) between perceived functional ability and perceived health, and between perceived functional ability and 
overall physical self-worth Also in elderly participants.

Finally present findings showed evidence for the existence of the gender and age differences reported in the literature as significant statistical differences were found for strength $(\mathrm{p}<.05$; Cohen's $d=.274)$ between male and female elderly participants from the total sample, revealing a small effect size of gender over physical strength with males presenting higher means score values than females. Further significant statistical differences were also found for physical confidence $(\mathrm{p}<.05$; Cohen's $\mathrm{d}=-.460)$, body $(\mathrm{p}<.05$; Cohen's $\mathrm{d}=-.411)$ and physical selfworth ( $\mathrm{p}<.05$; Cohen's $\mathrm{d}=-.506$ ) between male and female elderly participants in the age group of 65-75 yrs., revealing a moderate effect size of gender over the three physical self-subscales. In the present sample females reported higher mean score values than males contradicting the results presented by Sonstroem, Speliotis and Fava (1992) that showed higher mean score values for males in all subdomains.

\section{CONCLUSION}

Psychometric properties of the Physical Self Perception Profile analysed in the present study seem to indicate that other dimensions of the physical self may emerge as more relevant for elderly's self-perceptions in the physical domain such as a strong health concern, with elderly participants actively pursuing health and functional goals rather than personal feelings about sport competence and stamina. Many participants highlighted the functional dimension of their bodies as something very important for the way they perceived themselves and for their perceived health status. Therefore, present results provide evidence to discourage the use of this questionnaire as a reliable and valid instrument to assess physical self-perceptions in elderly groups.

Present findings are important as they provide further evidence for the differences on self-perceptions over the life span and should be analysed in further detail as they support the existence of other sub-domains related to health and functioning that may play a more important role to explain personal feelings about physical self in older people, suggesting the need for the development of a new instrument to assess the physical selfperceptions in elderly people.

\section{REFERENCES}

Bordens, K.S., \& Abbott, B.B. (2008). Research Design and Methods: A process approach (7th Edition). New York: McGraw - Hill Companies, Inc.

Button, E. J., Sonuga-Barke, E. J. S., Davies, J., \& Thompson, M. (2011). A prospective study of self-esteem in the prediction of eating problems in adolescent schoolgirls: Questionnaire findings. British Journal of Clinical Psychology, 35(2), 193-203.

Chase, L.A. (1991). Physical Self-perceptions and activity involvement in the older population. Dissertation Abstracts International, 52, 2458.

Cronbach, L. J. (1951). Coefficient alpha and the internal structure of tests. Psychometrika, 16, 297-334.

Department of Health (2004). At least five a week: evidence of the impact of physical activity and it's relationship to health. A report from the Chief Medical Officer. Department of Health, London.

Elavsky, S., McAuley, E., Molt, R., Konopack, J.F., Marquez, D.X., \& Hu, L. (2005) Physical Activity Enhances Long-Term Quality of Life in Older Adults: Efficacy, Esteem and Affective Influences. Annals of Behavioral Medicine, 30(2), 138-145.

Emery, E.M., McDermott, R.J., Holcomb, D.R., \& Marty, P.J. (2009). The Relationship Between Youth Substance Use and Area-Specific Self-Esteem. Journal of School Health, 63(5), 224-228.

Ferreira, J. P., \& Fox, K. R. (2007). An investigation into the structure, reliability, and validity of the Physical Self-Perception Profile in non-English spoken settings. International Journal of Applied Sport Sciences, 19(1), 25-46.

Ferreira, J.P., \& Fox, K.R. (2008). Physical Self-Perceptions and Self-Esteem in Male Basketball Players with and without Disability: A Preliminary Analysis using the Physical Self-Perception Profile. European Journal of Adapted Physical Activity. 1(1), $35-49$.

Fonseca, A., \& Fox, K.R. (2002). Como avaliar o modo como as pessoas se percebem fisicamente? Um olhar sobre a versão portuguesa do Physical Self-Perception 
Profile (PSPP). Revista Portuguesa de Ciências do Desporto. 5(2), 11-23.

Fox, K. R. (1990). The Physical SelfPerception Profile Manual. DeKalb, Il., Department of Physical Education, Office for Health Promotion: Northern Illinois University.

Fox, K. R. (2000). The effects of exercise on Self-perceptions and Self-esteem. In S. J. H. Biddle, K. R. Fox, \& S. H. Boutcher (Eds.), Physical Activity and Psychological Well-Being (pp. 88-117). London: Routledge.

Fox, K.R., Stathi, A., McKenna, J., \& Davis, M.G. (2007). Physical Activity and mental well-being in older people participating in the Better Aging Project. European Journal of Applied Physiology, 100, 591-602.

Fox, K. R., \& Corbin, C.B. (1989). The Physical Self-Perception Profile: Development of preliminary validation. Journal of Exercise and Sport Psychology, 11, 408-430.

Gothe, N.P., Mullen, S.P., Wójcicki, T.R., Mailey, E.L., White, S.M., Olson, E.A., Szabo, A.N., Kramer, A.F., \& McAuley, E. (2011). Trajectories of change in selfesteem in older adults: exercise intervention effects. Journal of Behavioral Medicine, 34, 298-306.

Harter, S. (1985). Competence as a dimension of self-evaluation: Towards a comprehensive model of self-worth. In R. Leahy (Ed.), The development of the self (pp. 55-121). New York: Academic Press.

Hu, L., \& Bentler, P.M. (1999). Cutoff criteria for fit indexes in covariance structure analysis: Conventional criteria versus new alternatives. Structural equation modeling, 6(1), 1-55.

Joreskog, K.J. (1969). A general approach to confirmatory maximum likehood factor analysis. Psychometrica, 34, 183-202.

Li, F., Harmer, P., Chaumeton, R., Duncan T.E., \& Duncan, S.C. (2002). Tai chi as a means to enhance self-esteem: A randomized controlled trial. Journal of Applied Gerontology, 21, 70-89

Mann, M., Hosman, C.M.H., Schaalma, H.P., $\&$ de Vries, N.K. (2004) Self-esteem in a broad spectrum approach for mental health promotion. Health Education Research, 19(4), 357-372.

McAuley, E., Blissmer, B., Katula, J., Duncan, T.E., \& Mihalko, S.L. (2000). Physical activity, self-esteem, and self-efficacy relationships in older adults: a randomized controlled trial. Annals of Behavioral Medicine, 22, 131-139.

McAuley, E., Elavsky, S., Motl, R.W., Konopack, J.F., Hu, L., \& Marquez, D.X. (2005). Physical activity, self-efficacy, and self-esteem: longitudinal relationships in older adults. The Journals of Gerontology. Series B, Psychological Sciences and Social Sciences, 60, 268-275.

McAuley, E., Konopack, J.F., Motl, R., Morris, K.S., Doerksen, S.E., \& Rosengren, K.S., (2006). Physical Activity and Quality of Life in Older Adults: Influence of Health Stats and Self-Efficacy. Annals of Behavioral Medicine, 31(1), 99-103.

Mirabel-Sarron, C., Vera, L., \& SamuelLajeunesse, B. (2001). Self-esteem and depression. Annales medicopsychologiques, 159(2), 144-148.

NIH Seniors Health (2010). Exercise and Physical Activity for Older Adults. National Institute on Aging. Retrieved February 24, 2010, from http://nihseniorhealth.gov/exerciseforoldera dults/toc.html

Opdenacker, J., Delecluse, C., \& Boen, F. (2009). The longitudinal effects of a lifestyle physical activity intervention and a structured exercise intervention on physical sel-perceptions and self-esteem in older adults. Journal of Sport and Exercise Psychology, 31, 743-760.

Ostrowsky, M. K. (2010) Are violent people more likely to have low self-esteem or high self-esteem? Aggression and Violent Behavior, 15(1), 69-75.

Rickli, R.E., \& Jones, C.J. (2001). Senior Fitness Test Manual. Champaign, Il: Human Kinetics.

Rosenberg, M. (1965). Society and the adolescent self-image. Princeton, NJ: Princeton University Press.

Semerjian, T., \& Stephens, D. (2007). Comparison Style, Physical SelfPerceptions and Fitness Among Older Women. Journal of Aging and Physical Activity, 15, 219-235.

Silverstone, P.H. (2011). Low self-esteem in different psychiatric conditions. British Journal of Clinical Psychology, 30(2), 185188.

Sonstroem, R.J., \& Morgan, W.P. (1989). Exercise and Self-esteem: Rationale and 
model. Medicine and Science in sport and Exercise, 21, 329-337.

Sonstroem, R. J., Speliotis, E.D., \& Fava, J.L. (1992). Perceived physical competence in adults: An examination of the Physical SelfPerception Profile. Journal of Sport \& Exercise Psychology, 14, 207-221.

Sonstroem, R.J., \& Potts, S.A. (1996). Life adjustment correlates of physical selfconcepts. Medicine and Science in Sports and Exercise, 28, 619-625.

Van der Vliet, P.,Knapen, J., Onghena, P., Fox, K.R., VanCoppenolle, H., David, A., Pieters, G., \& Peuskens, J. (2002). Assessment of physical self-perceptions in normal Flemish adults versus depressed psychiatric patients. Personality and Individual Differences, 32, 855-863.

U.S. Department of Health and Human Services (2001). Mental Health: Culture, Race, and Ethnicity - A Supplement to Mental Health: A Report of the Surgeon General - Executive Summary. Rockville MD: Department of Health and Human Services, Public Health Service, Office of the Surgeon General.

\section{Corresponding author:}

José P. Ferreira

E-mail address: jpferreira@fcdef.uc.pt 